\title{
ON THE STABILITY OF COUPLED OSCILLATIONS OF THE ELASTIC BOTTOM OF A RIGID RECTANGULAR CHANNEL AND IDEAL LIQUID
}

\author{
YURI KONONOV $^{1 *}$, ALEKCANDR LYMAR ${ }^{2}$ \\ ${ }^{1}$ Institute of applied mathematics and mechanics, \\ National Academy of Sciences of Ukraine, Sloviansk, Ukraine \\ ${ }^{2}$ Nikolaev National Agrarian University, Nikolaev, Ukraine
}

[Received: 04 October 2019. Accepted: 03 February 2020]

doi: 10.7546/JTAM.50.20.03.06

\begin{abstract}
Normal oscillations of the elastic bottom of a rigid rectangular duct with an ideal non-compressive fluid, which completely fills it, were investigated. The elastic bottom is a membrane. It is shown that the frequency equation is divided into two equations describing symmetric (even) and antisymmetric (odd) frequencies, and can be written in a single form for these frequencies. For even and odd frequencies, an approximate formula is obtained, from which approximate conditions follow for stability of coupled vibrations of an elastic basis and a fluid. These conditions are independent of the liquid height and the membrane mass. Exact stability conditions that coincide with hydrostatic conditions are derived. It is shown that the approximate value of the critical tension for asymmetric frequencies is $4 / 5$ times lower, and for symmetric frequencies, it is 0.818 times lower.
\end{abstract}

KEY WORDS: hydroelasticity, plane oscillations, ideal liquid, rectangular membrane, stability.

\section{INTRODUCTION}

Based on the unified Lagrangian approach, the problem of oscillations and stability of an elastic rectangular plate separating ideal liquids of different densities in a rectangular channel was first considered in the article [1]. In work [2] this problem was considered based on the Lagrange-Euler approach. The most complete study of free oscillations of a membrane on a free surface of a liquid in a rectangular channel was carried out in [3]. In [4] this problem was generalized in the case of a two-layer liquid with membranes on the free and internal surfaces for a rectangular channel with an elastic bottom. In work [5], transverse vibrations of a liquid in a long cylindrical container with a membrane or an elastic plate on a free surface were studied. Articles [6-8] continue the study of the publication [4]. For an infinitely long rectangular container with rigid walls Bauer \& Eidel [9] carried out a theoretical analysis on the

\footnotetext{
${ }^{*}$ Corresponding author e-mail: Kononov.yuriy.nikitovich@gmail.com
} 
following two systems: the first case is a tank with a rigid bottom and sidewall, where a free surface is covered by a membrane, while the second case is a container having a membrane bottom and a free surface. In the analysis, the nonlinear stress-strain relation and a large amplitude motion of the membrane were taken into consideration. In addition, some works has been performed on the hydroelastic behavior of separating walls in road and ship tankers [10], where tanks with fluids have finite sizes in all directions, only numerical approaches are available in the literature [11-13].

Many works deal with hydroelastic oscillations of an ideal liquid in circular and coaxial cylinders with rigid and elastic bases: [14-20] and many others. Symmetric and asymmetric vibrations of a plate or membrane on the free surface of an ideal fluid in a circular reservoir with an absolutely rigid bottom are considered in detail in [14]. Asymmetric oscillations of a circular plate on a free surface of an ideal liquid in a circular cylinder were studied in [15].

Regarding the non-linear problem of the topic, there are only a few studies in the literature $[9,16,17]$. In $[16,17]$, theoretical analysis and experimental study were carried out for nonlinear hydroelastic oscillations of a cylindrical tank with an elastic bottom. In paper [17], a linear axisymmetric free oscillations analysis of the bottom plate of the tank, coupled with the contained liquid is presented. This analysis takes into account the effect of an in-plane force in the plate due to the static pressure of the liquid.

In article [18], oscillations of an ideal fluid in a circular cylindrical tank with the same elastic bases in the form of circular plates are considered. The analytical method based on Fourier-Bessel expansion and the Rayleigh-Ritz method was proposed.

In the article [19], the frequency equation of axisymmetric oscillations of a heavy two-layer ideal liquid in a rigid annular cylindrical tank with an elastic top and bottom in the form of clamped annular plates is derived. The work [20] deals with the study of frequency equations of asymmetric and symmetric natural oscillations of an ideal bilayer liquid in a rigid circular cylindrical tank with an elastic top and bottom in the form of clamped circular plates. Using the example of a homogeneous liquid with a free surface and an elastic bottom in the form of a membrane, the frequency spectrum was analyzed analytically and numerically.

This article continues the research begun in [1-8]. The frequency equation of the coupled vibrations of a rectangular membrane and an ideal liquid is derived, the possibility of simplifying this equation is shown, approximate, and exact stability conditions of the coupled vibrations of the membrane and liquid are obtained.

\section{Formulation of the Problem}

Let us consider plane vibrations of an elastic base in the form of a membrane in a rigid rectangular channel with an ideal and incompressible liquid. The channel of the 
width $b(b=2 a)$ and height $h$ is filled with liquid of $\rho$ density. The coordinate system $O x y z$ is such that the plane $O x y$ lies on the undisturbed surface of the membrane, the axis $O y$ is directed along its middle of the channel, and the axis $O z$ is opposite to the acceleration vector of gravity $\vec{g}$ (Fig. 1).

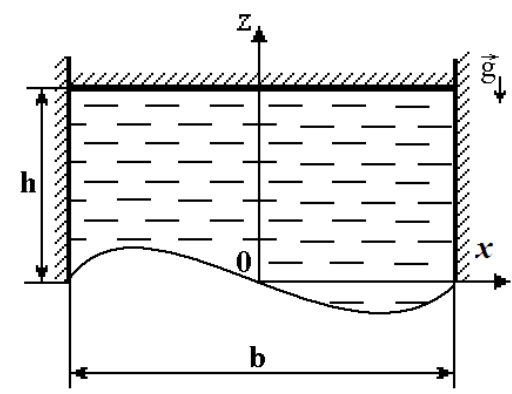

Fig. 1: A rigid rectangular channel with a liquid and an elastic bottom in the form of a membrane.

Oscillations of the membrane and liquid are considered in a linear formulation, assuming their oscillations without interruption, and the motion of a liquid is potential. Equations of plane vibrations of an elastic membrane and a liquid have the form $[4,6]$ :

(1) $k_{0} \frac{\partial^{2} W}{\partial t^{2}}-T \frac{\partial^{2} W}{\partial x^{2}}-g \rho W=\rho \frac{\partial \Phi}{\partial t}+Q+P_{0} \quad$ at $z=0, \quad \frac{\partial^{2} \Phi}{\partial x^{2}}+\frac{\partial^{2} \Phi}{\partial z^{2}}=0$ with boundary conditions

$$
\begin{gathered}
\left.\frac{\partial \Phi}{\partial x}\right|_{x= \pm a}=0,\left.\quad \frac{\partial \Phi}{\partial z}\right|_{z=h}=0, \quad \frac{\partial W}{\partial t}=\frac{\partial \Phi}{\partial z} \text { at } z=0, \\
\left.W\right|_{x= \pm a}=0, \quad \int_{-a}^{a} W d x=0 .
\end{gathered}
$$

Here $k_{0}=\rho_{0} h_{0} ; W(x, t), \rho_{0}, h_{0}$ are, respectively, normal flexure, density and thickness of the membrane; $\Phi(x, z, t)$ is the fluid velocity potential; $Q$ is an arbitrary time function; $P_{0}$ is a preset external pressure on the elastic bottom.

\section{Solution Method}

The function $\Phi(x, z, t)$ is represented in the form of the Fourier series with respect to Eigen-functions $\psi_{n}(x)$

$$
\Phi(x, z, t)=\sum_{n=1}^{\infty}\left[A_{n}(t) e^{k_{n} z}+B_{n}(t) e^{-k_{n} z}\right] \psi_{n}(x),
$$


where the functions $\psi_{n}(x)=\cos k_{n}(x+a)$ and their corresponding eigenvalues $k_{n}=\pi n / 2 a$ describe oscillations of an ideal liquid in the rectangular channel.

Considering (4) and (2), Eq. (1) takes the form

$$
k_{0} \frac{\partial^{2} W}{\partial t^{2}}-T \frac{\partial^{2} W}{\partial x^{2}}-g \rho W=-\sum_{n=1}^{\infty} \frac{a_{n} \ddot{W}_{n}}{k_{n}} \psi_{n}+Q+P_{0} .
$$

Here

(6) $W_{n}=\frac{1}{N_{n}^{2}} \int_{-a}^{a} W \psi_{n} d x, a_{n}=\rho \operatorname{coth} \kappa_{n}, N_{n}^{2}=\int_{-a}^{a} \psi_{n}^{2} d x=a, \kappa_{n}=h k_{n}$.

Thus, coupled oscillations of the elastic membrane and liquid are found from the system of integral differential Eqs. (5) and (6), the boundary conditions, and the incompressibility conditions of the liquid (3) and the given initial conditions.

\section{Normal Coupled Oscillations of the Elastic Membrane and LIQUID}

To find the Eigen-frequencies of the coupled oscillations of the elastic plate and the liquid, we set

$$
W(x, t)=w(x) e^{i \omega t}, \quad Q=\tilde{Q} e^{i \omega t}, \quad P_{0}=\tilde{P}_{0} e^{i \omega t} .
$$

Considering (7) we obtain the boundary-value problem

$$
\begin{gathered}
\frac{d^{2} w}{d x^{2}}+q w=-\frac{\omega^{2}}{T} \sum_{n=1}^{\infty} \frac{a_{n} w_{n}}{k_{n}} \psi_{n}+C, \\
w_{n}=\frac{1}{a} \int_{-a}^{a} w \psi_{n} d x, \quad \int_{-a}^{a} w d x=0,\left.\quad w\right|_{x= \pm a}=0,
\end{gathered}
$$

where

$q=\left(g \rho+k_{0} \omega^{2}\right) / T>0, C=-\left(\tilde{Q}+\tilde{P}_{0}\right) / T, \tilde{a}_{n}=a_{n}+k_{n} k_{0}, \tilde{d}_{n}=T k_{n}^{2}-g \rho$.

We look for the solution of Eq. (8) in the form of the general solution of the homogeneous equation and a particular solution of the inhomogeneous one

$$
w=\sum_{k=1}^{2} A_{k}^{0} w_{k}^{0}+\sum_{n=1}^{\infty} \tilde{C}_{n} \psi_{n}+w_{0} .
$$


Here $w_{k}^{0}=\{\sin p x, \cos p x\}, p=\sqrt{q}$, and $A_{k}^{0}, \tilde{C}_{n}, w_{0}$ are unknown constants.

We will represent $\tilde{C}_{n}$ and $w_{0}$ through unknown constants $A_{k}^{0}$. To do this, we substitute (10) into Eq. (8) and in the first relation (9)

$$
\tilde{C}_{n}=\omega^{2} \frac{a_{n}}{k_{n} d_{n}-\omega^{2} a_{n}} \sum_{k=1}^{2} A_{k}^{0} E_{k n}^{0}, \quad w_{0}=-\sum_{k=1}^{2} \tilde{w}_{k}^{0} A_{k}^{0},
$$

where $d_{n}=T k_{n}^{2}-g \rho-k_{0} \omega^{2}$,

$$
\begin{gathered}
E_{k n}^{0}=\frac{1}{a} \int_{-a}^{a} w_{k}^{0} \psi_{n} d x=\frac{T p}{a d_{n}}\left\{\left[(-1)^{n}-1\right] \cos \tilde{p},\left[(-1)^{n}+1\right] \sin \tilde{p}\right\}, \\
\tilde{w}_{k}^{0}=\frac{1}{2 a} \int_{-a}^{a} w_{k}^{0} d x=\frac{1}{\tilde{p}}\left\{\begin{array}{ll}
0, & k=1, \\
\sin \tilde{p}, & k=2
\end{array}, \quad \tilde{p}=a \sqrt{q} .\right.
\end{gathered}
$$

Taking into account the relations (11) and (10) the final expression for the shape of the flexure of the membrane will take the form

$$
w=\sum_{k=1}^{2}\left(w_{k}^{0}-\tilde{w}_{k}^{0}-\omega^{2} \sum_{n=1}^{\infty} \frac{a_{n} E_{k n}^{0}}{\omega^{2} \tilde{a}_{n}-k_{n} \tilde{d}_{n}} \psi_{n}\right) A_{k}^{0} .
$$

Here $\tilde{a}_{n}=a_{n}+k_{n} k_{0}, \tilde{d}_{n}=T k_{n}^{2}-g \rho$.

From the boundary conditions for membrane fixing, there are two linear homogeneous equations with respect to unknowns $A_{k}^{0}$

$$
\sum_{k=1}^{2}\left(B_{j k}-\omega^{2} \sum_{n=1}^{\infty} \alpha_{n} E_{k n}^{0} B_{j n}^{*}\right) A_{k}^{0}=0, \quad(j=1,2),
$$

where

$$
\begin{gathered}
B_{j k}=\left.\left(w_{k}^{0}-\tilde{w}_{k}^{0}\right)\right|_{x= \pm a}, \quad B_{j n}^{*}=\left\{\begin{array}{ll}
1, & j=1,(x=-a) \\
(-1)^{n}, & j=2,(x=a)
\end{array},\right. \\
\alpha_{n}=\frac{a_{n}}{\omega^{2} \tilde{a}_{n}-k_{n} \tilde{d}_{n}} .
\end{gathered}
$$

The frequency equation of natural oscillations of the elastic membrane and the liquid is obtained from the equality to zero of the determinant of the homogeneous system (14) 


$$
\begin{aligned}
\left(B_{11}-\omega^{2}\right. & \left.\sum_{n=1}^{\infty} \alpha_{n} E_{1 n}^{0}\right)\left(B_{22}-\omega^{2} \sum_{n=1}^{\infty} \alpha_{n} E_{2 n}^{0}(-1)^{n}\right) \\
& -\left(B_{12}-\omega^{2} \sum_{n=1}^{\infty} \alpha_{n} E_{2 n}^{0}\right)\left(B_{21}-\omega^{2} \sum_{n=1}^{\infty} \alpha_{n} E_{1 n}^{0}(-1)^{n}\right)=0
\end{aligned}
$$

Using the expansion of the functions $w_{k}^{0}$ in series with respect to complete and orthogonal system of functions $\psi_{n}(x)$, equation (16) can be rewritten as

$$
\left(\sum_{n=1}^{\infty} \beta_{n} E_{1 n}^{0}\right)\left(\sum_{n=1}^{\infty} \beta_{n} E_{2 n}^{0}(-1)^{n}\right)-\left(\sum_{n=1}^{\infty} \beta_{n} E_{2 n}^{0}\right)\left(\sum_{n=1}^{\infty} \beta_{n} E_{1 n}^{0}(-1)^{n}\right)=0 .
$$

Here $\beta_{n}=1-\omega^{2} \alpha_{n}=-k_{n} d_{n} /\left(\omega^{2} \tilde{a}_{n}-k_{n} \tilde{d}_{n}\right)$.

It follows from the expression $E_{k n}^{0}$ that Eq. (17) can be written in the form

$$
\left(\sum_{m=1}^{\infty} \tilde{\beta}_{2 m-1}\right)\left(\sum_{m=1}^{\infty} \tilde{\beta}_{2 m}\right)=0
$$

or

$$
\sum_{n=1}^{\infty} k_{n} /\left(\omega^{2} \tilde{a}_{n}-k_{n} \tilde{d}_{n}\right)=0
$$

Here $\tilde{\beta}_{n}=k_{n} /\left(\omega^{2} a_{n}-k_{n} d_{n}\right)=k_{n} /\left(\omega^{2} \tilde{a}_{n}-k_{n} \tilde{d}_{n}\right)$.

Thus, the frequency Eq. (17) splits into two equations describing odd ( $n=2 m-$ $1)$ and even $(n=2 m)$ frequencies and can be written in a single form for these frequencies (18). It should be noted that this simplification was achieved due to expansion of a function $w_{k}^{0}$ in series with respect to the complete and orthogonal system of Eigen-function $\psi_{n}(x)$. Normal modes of free oscillations of the membrane will be found from relations (13) and (14).

The left-hand part of Eq. (18) is a monotonically increasing function of the parameter $\omega^{2}$ on the interval $\left(k_{n} \tilde{d}_{n} / \tilde{a}_{n}, k_{n+1} \tilde{d}_{n+1} / \tilde{a}_{n+1}\right)(n=1,2, \ldots)$, the function takes on its values from $-\infty$ to $\infty$. Therefore, between two successive values $k_{n} \tilde{d}_{n} / \tilde{a}_{n}$ there is only one root of Eq. (18). This determines in advance the intervals, in which the natural frequencies are located. On the increase of number of terms of the series, the previous roots will be refined and new ones will appear. Squares of high frequencies $(n \gg 1)$ will be little different from the magnitude $\omega_{n}^{2}=k_{n}\left(T k_{n}^{2}-g \rho\right) /\left(\rho \operatorname{coth} \kappa_{n}+k_{n} k_{0}\right)$. It follows from this equality that $T>g \rho / k_{n}^{2}$, the addiction to $\omega_{n}^{2}$ depending on $T$ is linear, the greatest value $\omega_{n}^{2}$ 
will be when $k_{0}=0$, i.e. for the inertia-free membrane. Squares of frequencies $\omega_{n}^{2}$ are slightly dependent on the filling depth $h$ and decrease with its reduction. Thus, the problem under consideration has an infinite discrete spectrum of eigenvalues $\omega_{l}^{2}$, which are the roots of Eq. (18), and the corresponding Eigen-functions $w_{l}(x)$ form a complete orthogonal system of functions on the segment $[-a, a]$.

However, the problem under consideration has a number of physical features. In order that there is no rupture of continuity (formation of cavitation), the pressure inside the liquid must be non-negative. For this, the external pressure on the lower elastic foundation $P_{0}$ should not be less than the value $g\left(\rho h+k_{0}\right)$. This inequality does not take into account the magnitude of the membrane tension $T$. Naturally, by increasing the tension, one can always achieve positive pressure inside the liquid. Critical values of the tension will be found from the stability conditions of the vibrations of the membrane and liquid. The frequency Eq. (18) can be formally obtained from the equations of $[6,8]$ if we set the density of the lower liquid to zero $\left(\rho_{2}=0\right)$.

\section{Approximate and Accurate Conditions of Stability of Coupled Oscillations of the Membrane and Liquid. Dynamic ApProach}

If we retain two terms in the series of equation (18), then we obtain an approximate frequency equation, whose solution in dimensionless variables for odd $(n=1,3)$ and even $(n=2,4)$ frequencies, respectively, takes the form

$$
\begin{aligned}
& \Omega^{2}=6 \pi \frac{5 \pi^{2} \tilde{T}-1}{3 \operatorname{coth} \pi \tilde{h}+\operatorname{coth} 3 \pi \tilde{h}+6 \pi \tilde{k}_{0}}, \quad(n=1,3), \\
& \Omega^{2}=8 \pi \frac{10 \pi^{2} \tilde{T}-1}{2 \operatorname{coth} 2 \pi \tilde{h}+\operatorname{coth} 4 \pi \tilde{h}+4 \pi \tilde{k}_{0}}, \quad(n=2,4) .
\end{aligned}
$$

Here $\Omega^{2}=\omega^{2} b / g, \tilde{T}=T / g \rho b^{2}, \tilde{h}=h / b, \tilde{k}_{0}=k_{0} / \rho b$.

It follows from formulas (19) that the dependence of the square of a dimensionless frequency on a dimensionless tension is linear, the inertia-free membrane has the greatest frequency, frequencies almost do not depend on the filling depth at $\tilde{h}>1$ and decrease with decreasing depth.

From the inequality $\Omega^{2}>0$ we obtain the approximate stability conditions for the oscillations of the membrane and the liquid

$$
\begin{aligned}
& \tilde{T}>1 / 5 \pi^{2} \approx 0.025325 \quad(n=1,), \\
& \tilde{T}>1 / 10 \pi^{2} \approx 0.0123819 \quad(n=2,4) .
\end{aligned}
$$

The stability conditions (20) do not depend on the depth of liquid and the mass of the membrane. It can be seen from them that for the stability of asymmetrical oscillations, a twice as large amount of tension is needed than for symmetric ones. 
To clarify conditions (20), we proceed in the same way as in [6,8]. In Eq. (18) we set $\omega^{2}=0$ and solve the resulting equation with respect to the critical values of the parameters. When $\omega^{2}=0$, Eq. (18) takes the form $\sum_{n=1}^{\infty} 1 / \tilde{d}_{n}=0$ or in dimensionless variables

$$
\sum_{n=1}^{\infty} 1 /\left(n^{2}-\alpha^{2}\right)=0
$$

where $\alpha^{2}=1 / \tilde{T} \pi^{2}$.

Number series $\sum_{n=1}^{\infty} 1\left(n^{2}-\alpha^{2}\right)$ for odd $(n=2 m-1)$ and even $(n=2 m)$ values $n$ can be represented as

$$
\begin{aligned}
& \sum_{k=1}^{\infty} \frac{1}{(2 k-1)^{2}-\alpha^{2}}=\frac{\pi}{4 \alpha} \tan \frac{\pi \alpha}{2}, \\
& \sum_{k=1}^{\infty} \frac{1}{(2 k)^{2}-\alpha^{2}}=-\frac{1}{4 \alpha^{2}}\left(\pi \alpha \cot \frac{\pi \alpha}{2}-2\right) .
\end{aligned}
$$

The solution of Eq. (21) with $n=2 m-1$, taking into account (22), has the form $\alpha=2 l$. From this solution we find the critical values of the tension $\tilde{T}=1 / 4 \pi^{2} l^{2}$, which gives the following exact stability condition for $l=1$ :

$$
\tilde{T}>1 / 4 \pi^{2} \approx 0.02562 .
$$

The first root of Eq. (21) with $n=2 m$, taking into account (22), has the form $\pi \alpha / 2=4.493409458$, from which the updated stability condition follows:

$$
\tilde{T}>0.0127319 \text {. }
$$

Thus, the approximate value of the critical tension for asymmetric and symmetric frequencies is $4 / 5$ and 0.818 times lower, respectively. It should be noted that proximity of the approximate value and the exact takes place, taking into account two terms of the series we obtain sufficient accuracy for practice. 
6 Static stability Conditions for the Equilibrium Position of the Membrane AND Liquid. STATIC Approach

In the case of a static analysis of the perturbation problem, we have

$$
\begin{gathered}
\frac{d^{2} W}{d x^{2}}+\beta^{2} W=\tilde{C}, \\
\left.W\right|_{x=\mp a}=0, \quad \int_{-a}^{a} W d x=0,
\end{gathered}
$$

where $\beta^{2}=g \rho / T, \tilde{C}=-\left(P_{0}+Q\right) / T$.

Equation (23) has a solution

$$
W=A \sin \beta x+B \cos \beta x+C .
$$

Here $C=-\left(P_{0}+Q\right) / \rho g$.

The $Q$ constant is unknown. Substituting (25) into the boundary conditions and the incompressibility condition for the liquid (24), we obtain a homogeneous system of equations with respect to unknowns $A, B$ and $C$

$-A \sin \beta a+B \cos \beta a+C=0, A \sin \beta a+B \cos \beta a+C=0, B \sin \beta a+C \beta a=0$

or

$$
A \sin \beta a=0, \quad B \cos \beta a+C=0, \quad B \sin \beta a+C \beta a=0 .
$$

For existence of nonzero solution of the homogeneous system (26) its determinant must be equal to zero

$$
\sin \beta a(\beta a \cos \beta a-\sin \beta a) .
$$

It follows from (27) that the nonzero asymmetric solution $(A \neq 0)$ at $\beta a=\pi l$ and $B=C=0\left(Q=-P_{0}\right)$, a nonzero symmetric solution $(B \neq 0, C \neq 0)$ at $\cot \beta a=1 / \beta a$ and $A=0$.

From the equality $\beta a=\pi l(\beta a=\pi \alpha / 2)$ we get $T=g \rho a^{2} / \pi^{2} l^{2}\left(\tilde{T}=1 / 4 \pi^{2} l^{2}\right)$ and the asymmetric deflections of the membrane have the form $W_{l}=A_{l} \sin \pi l x / a$, from equation $\cot \beta a=1 / \beta a$ we have $T=g \rho / \beta_{l}^{2}\left(\tilde{T}=1 /\left(2 \beta_{l} a\right)^{2}, \beta_{l} a\right.$ are the root of this equation) and the symmetrical deflections of the membrane are written as $W_{l}=B_{l}\left(\cos \beta_{l} x-\cos \beta_{l} a\right)$.

These equilibrium modes are realized when the tension is not less than the corresponding critical values $g \rho a^{2} / \pi^{2}$ and $g \rho / \beta_{l}^{2}$. It can be seen that with an increase in 
the density or the channel width, the tension necessary for stability should increase. The pressure in a liquid should be positive for $z=0$, and taking into account the mass of the membrane, the pressure $P_{0}$ must be at least $g\left(\rho h+k_{0}\right)$.

It should be noted that the critical stress values found according to the static approach coincide with the exact values obtained by the dynamic approach. Thus, the simple static approach makes it possible to determine the conditions for the stability of coupled oscillations of a membrane and a liquid.

\section{Summary AND DiRECTIONS FOR FURTHER RESEARCH}

Based on the analytical and numerical studies, we can conclude the following:

1. Natural oscillation frequencies of the membrane and liquid for asymmetric and symmetric frequencies are presented in a single form.

2. An approximate formula for high frequencies is obtained. The dependence of the square of the frequency on the tension is linear. The highest frequency value will occur for an inertia-free membrane. Frequencies decrease with the decrease of the liquid depth.

3. Taking into account two terms of the series, an approximate formula is obtained for a square of dimensionless frequency. The main conclusions from this formula coincide with the results obtained for high frequencies.

4. The approximate stability conditions for the coupled vibrations of the membrane and liquid are derived. These conditions are independent of the depth of the liquid and the mass of the membrane. The stability of asymmetric oscillations requires twice as much critical tension as for symmetric vibrations.

5. The stability conditions of the static approach coincide with the exact stability conditions of the dynamic approach.

6. It follows from the exact stability conditions, that approximate values of the critical tension for asymmetric and symmetric frequencies are underestimated by $4 / 5$ and 0.818 times, respectively. Thus, taking into account two terms of the series gives accuracy sufficient for practice.

7. Using numerical calculations we establish that with the increase of the number of terms in series of the frequency equation the previous frequencies are refined and new ones appear. To obtain acceptable accuracy, it is enough to take into account 10-20 terms of the series.

In the future, it is planned to consider an elastic base in the form of a rectangular plate taking into account tensile or compressive stresses and to compare the stability conditions obtained from the dynamic and static approaches. It is also planned to consider this problem taking into account elastic upper and lower bases and to study the frequency spectrum and stability conditions. 


\section{ACKNOWLEDGEMENTS}

The study was carried out within the Fundamental Research Program of the Ministry of Education and Science of Ukraine, Project No. 0119U100042.

\section{REFERENCES}

[1] M.A. Ilgamov, Z.M. SaKhabutdinov (1974) On the stability of an elastic plate between liquids of different density, Selected problems of applied mechanics. Collection of articles for the seventieth birthday of Academician N. Chelomey, Moscow 341-346 (in Russian).

[2] Yu.N. Kononov, E.A. TAtarenKo (2002) Free vibrations of a two-layer liquid separated by an elastic plate in a rectangular channel. Theoretical and Applied Mechanics 36 170-176 (in Ukrainian).

[3] V.A. Trotsenko (1995) Free oscillations of a liquid in a rectangular channel with an elastic membrane on a free surface. Applied Mechanics 31(8) 74-80 (in Ukraine).

[4] Yu.N. Kononov, E.A. Tatarenko (2008) Free Vibrations of Elastic Membranes and Two-layer Liquid in a Rectangular Channel with an Elastic Bottom. Applied Hydromechanics $2008133-38$ (in Ukrainian).

[5] V.A. Trotsenko, R.I. Bogun (2009) Transverse vibrations of a liquid in a long cylindrical container with a membrane or an elastic plate on a free surface. Nonlinear oscillations 12(3) 379-404 (in Ukrainian).

[6] YU.N. Kononov, A.A. Lymar (2017) On the oscillation of a rectangular plate separating ideal liquids of different densities in a rectangular channel with one elastic base. Problems of computational mechanics and structural strength. Collection of scientific works 26 79-96 (in Ukrainian).

[7] YU.N. KonONOv, A.A. LYMAR (2017) Oscillations of a rectangular plate separating ideal liquids of different densities in a rectangular channel with elastic bases. News of the Zaporizhzhya National University. Physics and mathematics 20171 190-204 (in Ukrainian).

[8] YU.N. KonONOv, A.A. LYMAR (2018) On the update of the conditions of the stability of vibrations of the plate separating ideal liquids in a rectangular channel with hard foundations. Intern. Journal of Mechanical Engineering and Information Technology 6(1) $1755-1760$.

[9] H.F. BAUER, W. EIDEL (1988) Non-linear hydroelastic vibration in rectangular container. Journal of Sound and Vibration 125 93-114.

[10] H.F. BAUER (1981) Hydroelastic vibrations in a rectangular container. International Journal of Solids and Structures 17 639-652.

[11] Y.K. ChuenG, D. ZHOU (2000) Coupled vibratory characteristics of rectangular container bottom plate. Journal of Fluids and Structures 14 339-357.

[12] K.-H. Jeong, G.H. Yoo, S.C. Lee (2004) Hydroelastic vibration of two identical rectangular plates. Journal of Sound and Vibration 272 539-555. 
[13] D. ZHOU, W. LIU (2007) Hydroelastic vibrations of flexible rectangular tanks partially filled with liquid. International Journal for Numerical Methods in Engineering 71 149174.

[14] H.F. BAUER (1995) Coupled frequencies of a liquid in a circular cylindrical container with elastic liquid surface cover. Journal of Sound and Vibration 180(5) 689-704.

[15] K.-H. JEONG (2003) Free vibration of two circular plates coupled with bounded fluid. Journal of Sound and Vibration 260 653-670.

[16] M. CHIBA (1992) Non-linear hydroelastic vibration of a cylindrical tank with an elastic bottom, containing liquid; Part I: Experiment. Journal of Fluids and Structures 6 181206.

[17] M. CHIBA (1993) Non-linear hydroelastic vibration of a cylindrical tank with an elastic bottom, containing liquid; Part II: Linea axisymmetric vibration anaysis. Journal of Fluids and Structures 7 57-73.

[18] S. Tariverdilo, M. Shahmardani, J. Mirzapour, R. Shabani (2013) Asymmetric free vibration of circular plate in contact with incompressible fluid. Applied Mathematical Modelling 37(1-2) 228-239.

[19] Yu.. Kononov, V.P. Shevchenko, Yu.O. Dzhukha (2019) Axially symmetric vibrations of elastic annular bases and a perfect two-layer liquid in a rigid annular cylindrical vessel. Journal of Mathematical Sciences 240(1) 98-112.

[20] Yu.. Kononov, Yu.O. Dzhukha (2018) Oscillations of a two-layer ideal fluid in a rigid cylindrical tank with elastic bases. Nonlinear Oscillations 21(4) 496-513 (in Ukraine). 